\title{
Archéopages
}

Archéopages

Archéologie et société

Hors-série 3 | 2012

Nouveaux champs de la recherche archéologique

\section{L'investissement en archéologie au Royaume-Uni : expériences et perspectives}

\section{Martin Carver}

Traducteur : Madeleine Hummler

\section{OpenEdition \\ Journals}

Édition électronique

URL : https://journals.openedition.org/archeopages/454

DOI : $10.4000 /$ archeopages. 454

ISSN : 2269-9872

Éditeur

INRAP - Institut national de recherches archéologiques préventives

Édition imprimée

Date de publication : 1 janvier 2012

Pagination : $34-40$

ISSN : 1622-8545

Référence électronique

Martin Carver, "L'investissement en archéologie au Royaume-Uni : expériences et perspectives ».

Archéopages [En ligne], Hors-série 3 | 2012, mis en ligne le 01 janvier 2012, consulté le 24 février 2023

URL : http://journals.openedition.org/archeopages/454 ; DOI : https://doi.org/10.4000/archeopages. 454

Tous droits réservés 


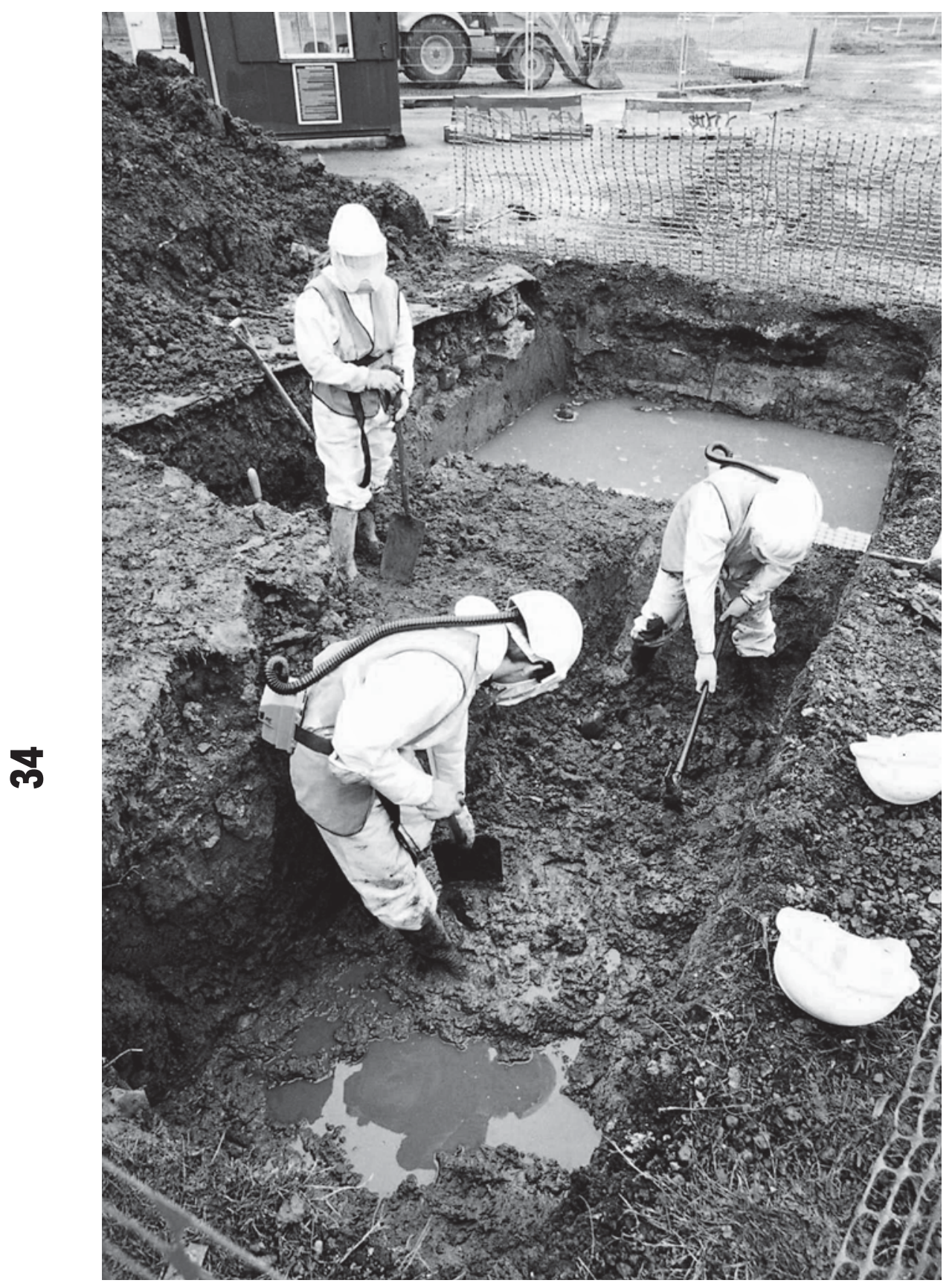

[Fig.1] Évaluation extrême : archéologues en vêtements de protection en train de tester des couches archéologiques

contaminées par des produits de

l'industrie chimique (FAS-Heritage $L t d)$. 


\section{L'investissement en archéologie au Royaume-Uni : expériences et perspectives}

\author{
Martin Carver ${ }^{\mathbf{1}}$ \\ Université de York (Royaume-Uni)
}

$\mathbf{L}$ e cadre législatif relatif à l'archéologie au Royaume-Uni reflète une longue tradition qui associe les vestiges du patrimoine culturel à la propriété terrienne, à moins qu'ils ne soient déclarés d'intérêt historique (listed), classés (scheduled) ou reconnus comme trésors d'État (treasure trove), ce qui leur confère un certain degré de protection. Les vestiges archéologiques non protégés, la grande majorité, peuvent être examinés dans le cadre d'un programme de recherche ou sous forme d'interventions en archéologie préventive. Dans les deux cas il est nécessaire d'obtenir la permission du propriétaire du terrain sur lequel ces vestiges se trouvent, étant donné qu'il est prima facie le propriétaire de ce patrimoine.

Le statut de l'archéologie au Royaume-Uni. Au dernier recensement, les activités d'archéologues employés dans le secteur de la recherche dépendent du Département de l'Innovation, des Universités et de la Recherche Scientifique (Department of Innovation, Universities and Science) qui finance également les organismes dédiés à la recherche sous l'égide du Département des Affaires, de l'Innovation et des Compétences (Department of Business, Innovation and Skills). L'archéologie préventive dépend des services responsables de l'aménagement du territoire qui sont sous la juridiction du Département des Collectivités locales (Department of Communities and Local Government). Cependant, ce sont les règlements relatifs à l'environnement, venant très souvent de l'Union Européenne, qui déterminent les raisons d'intervention et sont gérés par le Département de l'Environnement, de l'Alimentation et des Affaires rurales (Department of the Environment, Food and Rural Affairs). Pendant de longues années le ministère fut responsable de l'archéologie, et la réorganisation institutionnelle a entrainé une transmutation de notre patrimoine culturel vers la notion d'environnement historique. Les musées tombent sous l'égide du Département de la Culture, des Médias et du Sport (Department of Culture, méfiance. Ils travaillent pour des employeurs différents et reçoivent leurs salaires pour des fonctions différentes, suivant le rôle imputé à l'archéologie : source de recherche, atout local, élément du patrimoine historique ou quelque autre aspect relié de manière indirecte au tourisme, à l'aménagement du territoire, à la culture, aux médias ou au sport.

Les professions de l'archéologie. En pratique, les archéologues du Royaume-Uni travaillent au sein de trois secteurs principaux. Les fonctionnaires d'English Heritage sont responsables des monuments protégés et les fonctionnaires des administrations locales soutiennent les musées locaux et conseillent les aménageurs du territoire sur limpact archéologique de nouveaux aménagements. L'université héberge les archéologues engagés dans l'enseignement et la recherche. Dans le secteur privé (ou commercial) les entreprises offrent un éventail de plus en plus étendu de services, surtout en prospection, diagnostic et fouilles préventives ; il s'agit de "mitigation » (ou archéologie préventive) [Fig.1].

Dans la foulée de la politique entrepreneuriale promue par Margaret Thatcher, Tony Blair et maintenant David Cameron, le fonctionnariat se rétrécit. English Heritage, l'organisme d'État offrant le plus grand nombre de postes d'emploi, a connu une réduction massive de $32 \%$ dans son budget pour l'année en cours. Par contre, le secteur universitaire s'est agrandi de façon substantielle, alimenté par des fonds de recherche importants (provenant par exemple du Conseil de la Recherche Européenne ou de l'Arts and Humanities Research Council), par le système de prêts d'études introduit par le gouvernement socialiste de M. Blair. Ce système permet à n'importe quelle personne suffisamment qualifiée d'emprunter les fonds nécessaires pour subvenir à ses études, et de les rembourser une fois un certain niveau de salaire atteint. L'archéologie est un sujet d'étude très populaire, ce qui s'est avéré avantageux pour les universités qui enseignent cette discipline. La hausse récente des frais d'études a été commentée sévèrement par la presse, oubliant qu'elle avait soutenu ce système sous le gouvernement travailliste; on pourrait avancer qu'en pratique la différence n'est pas si grande car le remboursement des frais d'études est encore lié à un niveau de salaire ultérieur.

Cependant, c'est le secteur privé qui s'est développé le plus au cours des deux dernières décennies, pour une série de raisons. En premier lieu, le gouvernement a émis, en 1990, un document consultatif (PPG16, ou Planning Policy Guidance, $\mathrm{n}^{\circ} 16$ ), qui oblige toutes les administrations locales à réaliser un diagnostic sur l'impact qu'un nouvel aménagement pourrait avoir sur les vestiges archéologiques touchés. Étant donné qu'on peut envisager l'Angleterre comme un immense site archéologique, il s'ensuit que presque chaque fois qu'on remue le sol, l'archéologie se trouve impliquée, ce qui signifie que le diagnostic recommande une fouille et des relevés précis avant construction, processus dénommé précisément «mitigation». En deuxième lieu, les édifices encore sur pied font English Heritage à travers le ministère du Tourisme et du Patrimoine. Au Pays de Galles et en Écosse, les ministères décentralisés se partagent la responsabilité de la gestion du patrimoine. Ce ne sont donc pas moins de six ministères qui sont responsables pour l'autorisation, et indirectement le financement, de l'archéologie au Royaume-Uni. Il est donc bien excusable d'être désorienté ! Il en découle, par exemple, que des archéologues autrefois compagnons d'études, mais ensuite employés dans des secteurs différents, se rencontrent à peine (sauf lors de rares congrès) et commencent à se regarder avec une certaine 
également partie du patrimoine culturel et bénéficient de mesures semblables, chaque fois qu'on propose de transformer un bâtiment d'intérêt historique (PPG15). Troisièmement, du fait que l'emprise de l'État se rétrécit, toute une série de services autrefois fournis par l'État se voit maintenant assurée par le secteur privé. Ces services comprennent, entre autres, le conseil des aménageurs du territoire, la conservation et la présentation des monuments au public, la construction et la commercialisation dans les musées, la conception des expositions et la production de publications de plus en plus diverses. Par conséquent, les entreprises travaillent pour une multitude de maîtres d'ouvre, y compris les propriétaires, les trusts, les associations locales, qui, à leur tour, peuvent avoir recours à la loterie nationale (Heritage Lottery Fund) pour solliciter des fonds. Enfin, en quatrième lieu, certains pays en dehors du Royaume-Uni ont vu l'occasion d'employer des équipes britanniques, capables de fournir des services archéologiques sur contrat sans avoir la responsabilité du personnel.

Le nouvel acteur dans le secteur privé qui a pris le plus d'importance est le conseiller (ou consultant), un indépendant travaillant pour le compte de l'État, d'une collectivité locale ou d'un musée. Mais la plupart sont engagés par les promoteurs, collaborant avec d'autres consultants (architectes, conseillers en aménagement du territoire, juristes, etc.) autour d'un projet d'aménagement. Étant donné qu'ils sont engagés par ces promoteurs, le rôle des consultants en archéologie se résume à concevoir des interventions archéologiques qui allient un rendement maximum à un coût minimum.

Le secteur commercial se divise donc en deux: les consultants, dont le rôle est d'accommoder les exigences de la mitigation et les entreprises (autrefois les units) payées pour sa mise en œuvre. Comme le coût de l'opération est convenu avec le consultant, il existe une certaine tension entre les deux branches de la profession. Les meilleurs conseillers se comportent comme les inspecteurs d'État de jadis, faisant de leur mieux pour promouvoir l'archéologie et tenir tête aux promoteurs toujours motivés pour diminuer les coûts. Les pires professionnels sont motivés par leurs propres honoraires et n'exigent que le minimum possible en opération archéologique.

L'Écosse et le Pays de Galles sont restés attachés plus longtemps que l'Angleterre à l'esprit socialiste, ce qui leur a permis de soutenir des agences nationales (CADW au Pays de Galles, Historic Scotland en Écosse). Mais la déréglementation est en cours, ce qui signifie que des entreprises privées, certaines venant d'Angleterre, opèrent maintenant sur leur territoire.

Les problèmes à court terme. Les problèmes à court terme sont évidents. Les archéologues travaillant dans le secteur privé sont soumis aux fluctuations du marché, qui est actuellement extrêmement volatile. À l'issue de la construction de l'autoroute N1 par le gouvernement irlandais, des centaines d'archéologues ont été licenciés, y compris un grand nombre de Polonais que la demande en main d'œuvre avait attiré. Les gouvernements du Royaume-Uni et d'Irlande ont également supprimé une série de projets d'infrastructure dans l'espoir de réduire les dettes nationales et de faire impression sur le marché des obligations. La suppression de 10 millions de livres sterling dédiées au nouveau centre d'accueil de Stonehenge figure parmi les gestes les plus futiles; il s'agit d'une somme relativement modique, qui représente une petite part de l'ensemble, financé par d'autres sources. Le résultat a été, non d'arrêter la remise en état de Stonehenge (beaucoup considèrent sa négligence comme un véritable scandale national), mais de ralentir ses progrès et de donner l'impression que le nouveau gouvernement de coalition se désintéresse du patrimoine. Ce gouvernement risque d'aller plus loin, en assouplissant les contraintes auxquelles les aménageurs sont soumis et en relâchant les critères de la mitigation archéologique. On se rappellera que l'archéologie préventive n'est pas soumise à une loi, seulement à un document consultatif, ce qui permet de faire machine arrière assez facilement. En effet, en 2011, un élu municipal du Cambridgeshire a annoncé qu'il avait l'intention de se passer de toute intervention archéologique, qualifiant ceux qui s'y opposeraient de «bunnyhuggers» (« embrasseurs de lapins »!). Cet incident, rapidement baptisé de «Bunnygate», a provoqué une réaction rapide et efficace, mais les archéologues au Royaume-Uni restent sur le qui-vive.

Si la crise économique continue, il est bien probable qu'elle entrainera un manque d'archéologues qualifiés, d'abord au chômage et ensuite absorbés par d'autres professions. Tout naturellement c'est une cause de préoccupation majeure pour les archéologues, mais ce n'en est qu'une parmi d'autres. Dans sa récente thèse de doctorat, Paul Deverill (maintenant chargé de cours au King Alfred's College, à Winchester) a analysé les réponses de plus d'un millier d'archéologues salariés à un questionnaire destiné à évaluer leur bien-être, leur moral et l'idée qu'ils se font de l'avenir. Une partie des réponses était prévisible, autour des salaires insuffisants et du climat anglais. D'autres observations étaient plus inattendues : le manque de direction aurait un effet négatif sur la main d'œuvre; les responsables de chantier manqueraient de compétences pour diriger une équipe; ils démoraliseraient la main d'œuvre et ils dévalorisaient systématiquement les vestiges archéologiques afin de respecter le budget et les délais régissant la survie de leur propre emploi. Les responsables de chantier seraient employés pour gérer (alors qu'ils ne sont pas qualifiés en gestion) et négligeraient l'archéologie (alors qu'ils sont qualifiés en cette matière). La raison la plus courante citée par ceux qui envisageaient de quitter la profession est encore plus révélatrice : ils auraient décidé d'abandonner parce que leur poste ne leur permettrait plus de s'engager dans une recherche qui les avait passionnés lors de leurs études. Les raisons d'entreprendre des fouilles préventives n'incorporent aucune obligation de comprendre ou d'étudier les sites, seulement d'en faire le relevé le plus rapidement possible en utilisant des protocoles répétitifs et normatifs. Les archéologues éprouvent 
le sentiment qu'ils font davantage partie d'une sous-branche de l'industrie du bâtiment qu'ils ne sont acteurs de la recherche. Le résultat est une situation déjà esquissée plus haut : une fois les études terminées, les archéologues se retrouvent séparés par les emplois qu'ils occupent : quelquesuns décrochent un poste universitaire, quelques rares autres sont employés par l'État, et la grande majorité travaille dans le secteur privé. La mission et l'esprit sont entièrement différents dans chaque secteur, ce qui crée une faille de plus en plus large. Les occasions de transfert d'un secteur à l'autre se font de plus en plus rares ; en effet les archéologues eux-mêmes croient qu'ils pratiquent des métiers différents (en recherche, en gestion du patrimoine, en archéologie préventive) et ces diverses missions se trouvent reflétées dans les conditions d'emploi et les objectifs des employés.

L'éviction des acteurs bénévoles et les méthodes de fouille. En plus du schisme entre le secteur universitaire, le secteur commercial et le secteur national, les archéologues bénévoles, autrefois pilier central formé par une multitude d'associations locales, ont été exclus du processus, malgré les protestations auprès d'une succession de gouvernements qui manifestent un intérêt de pure forme pour la « communauté », le « régionalisme» ou encore la « diversité ». Quoique les associations locales continuent à se réunir, on ne leur demande pas leur avis au sujet de projets d'aménagement dans leur région, et les fouilles préventives sont souvent menées par des entreprises qui n'ont jamais travaillé dans la région et en ignorent presque tout. Cet état des affaires est dû en partie au fait que les contrats en archéologie préventive sont octroyés sur la base du moindre prix et une offre provenant de l'extérieur peut être celle qui est retenue ; les règlements concernant la santé et la sécurité au travail sont en partie évoqués pour justifier l'exclusion des bénévoles.

Un effet secondaire surprenant, causé par l'absence de bénévoles sur les chantiers, a été un mouvement subtil dans les techniques de fouille, qui se sont orientées vers un système américain de fouilles sur de petites surfaces plutôt que sur les grandes, comme c'était autrefois la norme. Comme nous le savons bien, il est souvent nécessaire de décaper une grande surface, visible dans sa totalité et de haut, afin de comprendre l'agencement d'un site. Mais bien sûr, une telle surface ne peut être décapée que si l'on dispose d'un grand nombre de fouilleurs. Si tous ces fouilleurs étaient payés, le coût serait trop élevé. Par conséquent, les entreprises conçoivent leurs projets non pour servir les besoins de l'archéologie, mais pour pouvoir retenir leurs employés, du moins ceux bénéficiant d'un contrat à durée indéterminée. Le résultat est une multitude de tranchées et de « caissons », au détriment de la recherche archéologique.

Un autre problème d'ordre technique surgit: les méthodes scientifiques utilisées sur un site archéologique ont évolué de façon de plus en plus raffinée au cours des dernières décennies. Nous disposons maintenant de techniques microscopiques, pour étudier les restes de végétaux et d'insectes comme données indirectes sur le milieu, et de nano-techniques qui éclairent le type d'activité sur un site, par la lecture de données chimiques et physiques, y compris les traces d'ADN. Ces techniques ont révélé des détails surprenants de traces d'activité dans une structure ou même au niveau d'un paysage entier. Mais, bien sûr, l'emploi de ces techniques est non seulement coûteux, mais exige aussi une approche qui diffère des anciennes normes : la routine, qui consiste à enregistrer des unités stratigraphiques (single context) et à construire un diagramme stratigraphique, ne fournit qu'une fraction des données qui pourraient être enregistrées et empêche même une analyse plus raffinée en décapant une couche à la fois.

La conception de projets archéologiques et les publications. Notons tout de même qu'English Heritage (fondé en 1982) a fait figure de pionnier dans la conception de projets en archéologie. Cet organisme d'État a été parmi les premiers à exiger un diagnostic et la préparation ainsi que la promulgation d'un dossier de projet complet (project design) avant le début d'une intervention (une procédure inaugurée par le projet de recherche de Sutton Hoo en 1983). De plus, English Heritage a subventionné la préparation de ces dossiers et a encouragé le secteur privé à faire de même. Cette façon de procéder n'a malheureusement pas encore été entièrement adoptée par le secteur universitaire, bien que ce dernier reçoive des sommes importantes de la part des conseils de recherche, qui n'exigent pas de diagnostic ou de dossier de projet préalables.

Le principe de dossier préalable s'étend aussi aux programmes d'analyses et de publications ; English Heritage exige, et finance souvent une évaluation de ces étapes. Étant donné que les organes responsables de l'archéologie au RoyaumeUni ainsi que les intérêts qu'ils représentent sont très divers, il n'est pas étonnant que les formes de publications le soient également, voire quelquefois contradictoires. Dans le secteur universitaire, le fait que les grands fonds de recherche soient souvent étalés sur un période de cinq ans entraine un certain retard dans les publications, qui sortent sous forme de monographies importantes; mais le système d'évaluation de la recherche exige une production rapide d'articles (au moins quatre sur une durée de quatre ans dans le système en vigueur). Ces articles ont un impact maximum si on réussit à les placer dans des revues de haut niveau (par exemple Antiquity), mais ces dernières demandent presque toujours des articles concis (500o mots dans le cas d'Antiquity). Ce cycle, et ce genre de publication, n'est pas adapté aux recherches de terrain, qui exigent souvent une dizaine d'années pour mener à terme un projet d'envergure qui comprend aussi la diffusion d'une quantité importante de données. En pratique, les quelques universitaires qui fouillent doivent publier leurs résultats à plusieurs niveaux : rapports de fouilles monographiques illustrés, articles théoriques, synthétiques ou réflexifs

\section{के}




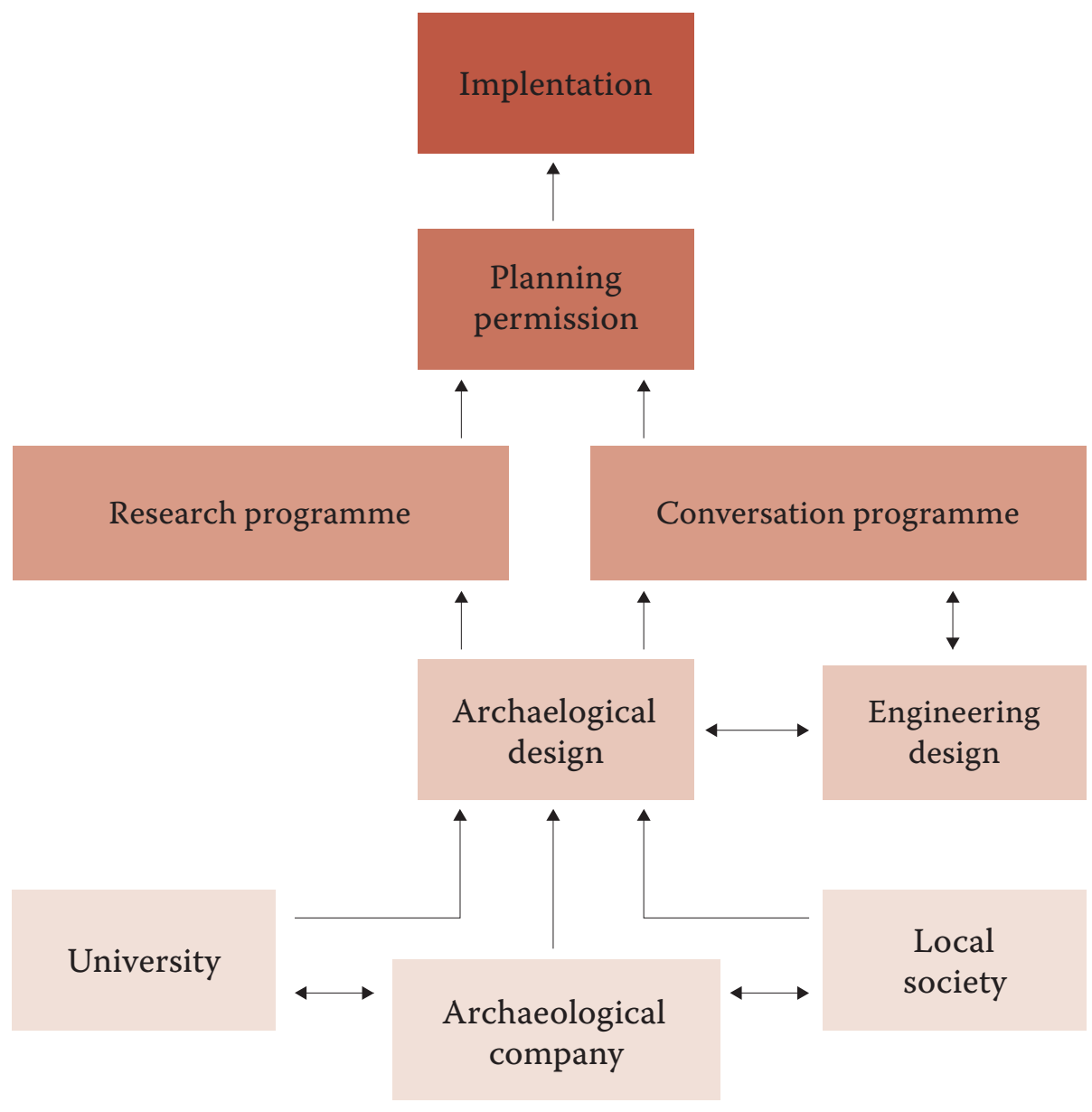

[Fig.2] Une approche intégrée combinant les objectifs de la recherche, de la conservation et des collectivités

locales. 
dans des revues à haut impact. S'ils travaillent à l'étranger, ils ne sont pas obligés de publier dans les organes ou dans la langue de ce pays.

Le secteur commercial a mauvaise réputation en ce qui concerne la publication, ce qui n'est peut-être pas entièrement mérité. Une entreprise archéologique honore son contrat avec le promoteur (le client) et son premier (en fait, son unique) devoir est de produire un rapport pour ce client (client report) ; le contenu de ce rapport est en grande partie déterminé par les autorités locales responsables de l'aménagement du territoire. L'entreprise ne reçoit rien pour étudier les sites fouillés et toute étude effectuée est faite pour le compte des entreprises qui doivent investir en temps et en argent, ce dont elles manquent en général. Ajoutons que, assez souvent, les projets commerciaux ne contribuent que très peu à la recherche car ils n'ont pas été conçus dans un tel but. Ce qu'ils produisent, ce sont des relevés, des analyses stratigraphiques et du mobilier, en somme: des données. L'Archaeological Data Service, conçu à l'origine comme dépôt de données, dédié à la conservation des données numériques, mais qui s'est aussi développé en service d'archives pour les rapports de fouilles menées par le secteur commercial, offre peut-être une solution aux chercheurs, s'ils sont capables d'utiliser ces ressources.

Comme nous l'avons indiqué, le secteur commercial est bien conscient d'avoir peu de possibilités pour mener à bien un programme de recherche, et encore moins de le publier. Les plus grandes entreprises affrontent le problème en utilisant leurs profits (et quelquefois en faisant appel au mécénat des entreprises de construction) afin de produire de grandes monographies bien illustrées dont le contenu est un véritable instrument de recherche (par exemple, une collaboration entre Wessex et Oxford sur les fouilles de l'aéroport de Stanstead). Ces travaux offrent une satisfaction aux employés et rehaussent le statut des entreprises. Ayant ainsi acquis un profil plus élevé, et accru leurs profits, les plus grandes entreprises rachètent les plus petites comme toute autre entreprise dans un système capitaliste. Cette tendance risque de s'accentuer dans un période de manque de liquidités, où les plus faibles périssent.

Les perspectives. Les problèmes que nous avons évoqués sont liés à l'organisation de l'archéologie d'une nation, mais pas exclusivement. À première vue, une profession soutenue par son gouvernement peut espérer de meilleures conditions pour ses employés. Mais ceci n'a pas été le cas dans les agences plus centralisées du Pays de Galles et d'Écosse. Les employés n'ont toujours pas la possibilité de poursuivre un thème de recherche et on leur demande d'opérer au sein de bureaucraties suffocantes. Les agences ne disposent pas de ressources suffisantes pour monter des interventions à grande échelle, sont réticentes à tenter plus que le strict nécessaire et négligent ainsi les possibilités de résoudre des questions posées par la recherche. De plus, comme indiqué plus haut, leurs budgets peuvent être réduits sans raison ni délai pour des raisons étrangères à l'archéologie.

Les questions les plus pertinentes à poser à chaque maître d'œuvre et à chaque archéologue sont les questions qui ont trait à l'archéologie en général, quelles que soient les raisons et quels que soient les objectifs qui exigent une intervention archéologique; des questions que l'État, les promoteurs tout aussi bien que le public devraient poser : pourquoi payons-nous? Quelle raison s'impose pour une intervention archéologique? Quel est le produit de ce travail ? Les réponses n'apportent pas nécessairement des éléments utiles pour le patrimoine, la fierté locale (éléments non mesurables), ou pour le tourisme. Tous ces avantages ne sont que les sous-produits d'un produit principal : l'acquisition de nouveaux savoirs. En savoir plus sur le passé, c'est notre mission bien simple; que ce soit sur un oppidum, désert et protégé, ou en plein centre-ville, nous n'avons qu'une seule chance. Nous devons donc investir le maximum d'efforts dans la formulation d'un projet en utilisant toutes les ressources de la prospection non invasive à notre disposition. Cette conception de projet (project design) doit inclure un programme de recherche, un autre de conservation, un troisième de mitigation et un dernier de présentation au public. Tout l'art consiste à équilibrer le mieux possible ces exigences, pour que les buts puissent se complémenter. Mettre toutes nos compétences en œuvre, c'est faire notre devoir de professionnels.

Si nous réussissions à nous accorder entre archéologues, nous pourrions oublier nos différends. Chaque projet, qu'il soit essentiellement de recherche, de conservation ou de mitigation, pourrait compter sur la participation du secteur universitaire, de l'État, du secteur privé et des communautés locales [Fig.2]. Nous aurions enfin une profession intégrée. De plus, nous aurions, au sein des professions alliées, redéfini notre statut en tant que spécialistes et non plus comme ouvriers manuels. Si les interventions archéologiques au Royaume-Uni au cours des années à venir, exécutées surtout par le secteur privé, tiennent à gagner de l'envergure, à recevoir des fonds suffisants et à donner aux archéologues le sentiment du travail bien fait, il nous faut changer radicalement notre façon d'opérer et de négocier. Légiférer n'est pas la solution. Nous devons prendre notre place aux tables de négociations, avec d'autres conseillers dont le rôle est de rehausser la valeur d'un projet d'aménagement. Nous nous trouverons en face d'architectes, auxquels nous ressemblerons de plus en plus dans notre manière d'agir avec créativité, et gagnerons le même respect et des honoraires similaires. Nous ne nous contenterons plus de sauvegarder les vestiges archéologiques ou de minimiser les dommages, mais contribuerons à augmenter la valeur d'un projet par nos concepts créatifs (Carver, 2011).

Les gouvernements successifs continueront à débattre des mérites ou non de l'entreprise privée ou de la redistribution sociale. Peu d'archéologues ont l'oreille du gouvernement britannique; il nous incombe donc de nous adapter afin d'augmenter 
au maximum nos chances de survie en tant que professionnels et de mettre au maximum à profit la pérennité et la reconnaissance publique de nos ressources archéologiques. Notre arme principale n'est pas la législation, mais la recherche et sa promulgation dans toutes les couches de la société.

Barver M., 2011 : Making Archaeology Happen : Design versus Dogma, Walnut Creek (CA), Left Coast Press.

\section{L'archéologie préventive suisse}

Gilbert Kaenel

Musée cantonal d'archéologie et d'histoire de Lausanne

D ans les différents bilans consacrés aux apports de l'archéologie préventive en Europe ou dans le monde, le cas de la Suisse est très rarement pris en compte. Cela tient sans doute à l'exiguïté de son territoire, comparable grosso modo à la Bourgogne (et encore faudrait-il déduire les montagnes au-dessus de 2 à $3000 \mathrm{~m}$ et les plans d'eaux...), mais aussi au fonctionnement même de son ou, plutôt, de ses archéologies.

\section{Quelques fondamentaux en guise de} préliminaire. La constitution de l'État fédéral de 1848 attribue à la Confédération helvétique un rôle subsidiaire à l'égard des «sites évocateurs du passé et des monuments d'importance nationale»; on lit en effet, dans le même article, avant que le terme «archéologie» ne s'impose, que «la protection de la nature et du paysage relève du droit cantonal ». En d'autres termes, c'est comme si, pour reprendre notre comparaison avec la Bourgogne, 26 services archéologiques ( 23 cantons +3 demi-cantons) étaient souverainement responsables de la gestion de leur patrimoine archéologique. On ajoutera un article du code civil suisse (entré en vigueur le $1^{\mathrm{er}}$ janvier 1912), précisant que « les antiquités qui n’appartiennent à personne et qui offrent un intérêt scientifique sont la propriété du canton sur le territoire duquel elles ont été trouvées ». Formulation d'une efficacité redoutable, à l'image de sa simplicité angélique, qui règle, sans appel, la propriété des trouvailles archéologiques (et donc les problèmes liés à leur dévolution), tout en affirmant que la responsabilité de conservation et de restauration de ce patrimoine incombe aux cantons, à charge pour ces derniers de s'organiser et de confier ces missions à des musées.

Dernier acte, que nous qualifions de petit «miracle»: un arrêté fédéral du 13 mars 1961 précise le rôle subsidiaire de la Confédération dans le cadre des travaux dont elle a la maîtrise de l'ouvrage, en particulier des autoroutes : «Les frais de fouilles pour la recherche d'antiquités sur le tracé de futures routes nationales, de déblaiement ou des levés scientifiques des trouvailles (photographies, esquisses, mensurations) sont des frais de construction des routes nationales. La Confédération participe à ces frais, par prélèvement sur les crédits accordés pour les routes nationales ». Il y a donc un peu plus de 50 ans que le principe même de la future «Convention de Malte» a été introduit en Suisse, laquelle a initié des opérations d'archéologie «préventive» avant la lettre, sans le savoir...

Entre « lacustres » et arrière-pays. Mais trêve de réflexions politico-administratives, voyons quelques résultats choisis, en Protohistoire, en Suisse occidentale, à l'est du Jura et jusqu'au pied des Alpes ; les derniers cantons dans lesquels l'aménagement du réseau autoroutier (du rail également) a été conduit, ont donc très largement bénéficié des avancées méthodologiques des trente à quarante années écoulées, mais aussi de questionnements scientifiques plus ambitieux, liés à des opérations dotées d'une manne fédérale également revue à la hausse.

Nous ne reviendrons pas sur le «boom » de l'archéologie des palafittes, dès la première intervention «moderne» en 1964 à Auvernier (canton de Neuchâtel) : la documentation exceptionnelle recueillie et élaborée, avec l'aide de nombreuses disciplines des sciences naturelles ou de l'environnement (dendrochronologie pour n'en évoquer qu'une), fournissent une image de plus en plus précise du développement des cultures du Néolithique moyen à la fin du Bronze final (environ 4300-80o avant notre ère) et du fonctionnement des sociétés.

Mais, au-delà de telles avancées saisissantes, la relation avec les terrasses et vallons situés au plus à quelques kilomètres des rives (où passent les autoroutes...) modifie radicalement cette image, aussi riche soit-elle, en réduisant un déséquilibre criant entre établissements lacustres et terrestres. D'une part les campagnes de l'âge du Bronze et de lâge du fer sont densément colonisées, ce que l'on n'osait pas postuler jusque-là en ces termes, et on y rencontre, d'autre part, en négatif pourrait-on dire (mais pas uniquement), le reflet des périodes qui ne sont pas représentées en milieu palafittique: le début du Bronze ancien (avant 1800 avant notre ère, pour simplifier), la plus grande partie du Bronze moyen et le début du Bronze final (environ 1500 à 1050 avant notre ère), le premier et le second âge du fer (dès 800 avant notre ère) après l'abandon définitif des installations villageoises sur les rives des lacs. Les recherches en cours renouvellent ainsi fondamentalement les perspectives en termes de dynamique du peuplement. Pour l'âge du fer, on ne recensait, «avant les autoroutes», pratiquement que des découvertes funéraires, tumuli hallstattiens, tombes dites «plates» du second âge du fer, et quelques sites au statut particulier, fortifications sur des hauteurs, voire résidences princières des $\mathrm{VI}^{\mathrm{e}}$ et $\mathrm{V}^{\mathrm{e}}$ siècles et, bien sûr, les oppida dès la fin $\mathrm{du} \mathrm{II}^{\mathrm{e}}$ siècle avant notre ère.

Prenons un exemple, les tronçons de l'autoroute A1 en terre fribourgeoise, au sud des lacs de Morat et de Neuchâtel : $23 \mathrm{~km}$ de tracé, 25 ans de fouilles (1975-200o), une centaine de sites de l'âge du Bronze et du fer, étudiés évidemment à des degrés différents. On peut transposer ce constat sur les autres tronçons autoroutiers, jusque dans le monde alpin, avec le site exceptionnel de Gamsen, au pied du Simplon (BrigGlis, Waldmatte, canton du Valais), fouillé entre 1987 et 1999: pas moins de 38 horizons stratigraphiques, 